\title{
THELOHANELLUS NJINEI N. SP. et T. LAGDOENSIS N. SP., MYXOSPORIDIES (MYXOZOA : MYXOSPOREA) PARASITES DeS SCHILBEIDAE et CithaRINIDAE (POISSONS télÉOSTÉENS) au Cameroun (Afrique centrale)
}

\author{
FOMENA A.*, FARIKOU-OUMAROU*, TANG II C.*. \& BOUIX G.**
}

Summary: THELOHANELLUS NIINEI SP. NOV. AND T. LAGDOENSIS SP. NOV, MyXosporidia (MyXozoa : MyXosporea) parasites of SCHILbeidae and Citharinidae fishes in Cameroon (Central Africa)

The study of Myxosporidia infecting Schilbe mystus (Linné, 1758) (Schilbeidae) and Citharinus citharus (Geoffroy Saint-Hilaire, 1809) (Citharinidae) from the Sanaga and Bénoue basins revealed the presence of two new species of the genus Thelohanellus kudo, 1993. These species are Thelohanellus niinei sp. nov. and Thelohanellus lagdoensis sp. nov., found in the gut of Schilbe mystus and Citharinus citharus respectively. New data are also given on the diagnosis of Thelohanellus citharini, a species previously described in Chad.

KEY WORDS : Myxosporidia, Thelohanellus, fish, Schilbeidae, Citharinidae, parasite, fresh water, Cameroon, Africa.

\section{Résumé :}

L'examen de Schilbe mystus (Linné, 1758) (Schilbeidae) et Citharinus citharus (Geoffroy Saint-Hilaire, 1809) (Citharinidae), capturés respectivement dans les bassins de la Sanaga à Edéa et de la Bénoué à Lagdo, a révélé la présence de deux espèces nouvelles de Myxosporidies appartenant au genre Thelohanellus Kudo, 1993 : Thelohanellus njinei n. sp., parasite de l'intestin de Schilbe mystus et Thelohanellus lagdoensis n. sp., parasite de l'intestin chez Citharinus citharus. Des informations complémentaires sont données sur la diagnose de Thelohanellus citharini, parasite antérieurement décrit au Tchad.

MOTS CLÉS : Myxosporidies, Thelohanellus, poisson, Schilbeidae, Citharinidae, parasite, eau douce, Cameroun, Afrique.

les Myxosporidies ont été réalisées dans les pays suivants : Bénin, Botswana, Burkina-Faso, Cameroun, Égypte, Ghana, Nigeria, Ouganda, Sénégal, Tchad et Tunisie. Dans ce continent, on recense aujourd'hui une centaine d'espèces décrites chez les poissons d'eau douce. Les espèces connues appartiennent aux genres Myxobolus Bütschli, 1882, Myxidium Bütschli, 1882, Myxobilatus Davis, 1944, Sphaerospora Thélohan, 1892, Parahenneguya Sakiti, 1997, Henneguya Thélohan, 1892, Chloromyxum Mingazzini, 1890 et Thelohanellus kudo, 1933.

En 1997, Fomena et Bouix ont donné une clé de détermination des Myxosporidies des poissons d'eau douce d'Afrique. Au Cameroun, les travaux portant sur les Myxosporidies sont surtout ceux de Fomena (1986, 1995), Fall et al., 2000, et traitent exclusivement des parasites des poissons d'eau douce. Les genres jusqu'à présent récoltés sont : Myxidium, Myxobolus, Sphaerospora, Chloromyxum, Henneguya et Thelohanellus. Le genre Thelohanellus est aujourd'hui représenté par trois espèces : Thelohanellus valeti, T. sanagaensis, T. assambai, toutes parasites des Cyprinidae des genres Labeo et Barbus.

Au cours d'une étude plus générale des Myxosporidies parasites des Téléostéens d'eau douce du Cameroun, nous avons trouvé deux espèces nouvelles dont nous donnons la description dans la présente note : Thelohanellus njinei $\mathrm{n}$. sp., parasite de Schilbe mystus (Schilbeidae), Thelohanellus lagdoensis n. sp. parasite de 
Citharinus citharus (Citharinidae). Des données nouvelles sont fournies sur la diagnose de Thelohanellus citharini Kostoïngué, Fall, Faye et Toguebaye, 1999, parasite de Citharinus citharus au Tchad.

\section{MATÉRIELS ET MÉTHODES}

I es poissons examinés ont étés récoltés au Cameroun (Afrique centrale) entre mai 2005 et mai 2006, notamment dans la retenue de Lagdo sur la Bénoué (province du Nord) et dans la rivière Lem bass, affluent de la Sanaga à Edéa (province du Littoral). Les captures ont été effectuées à l'aide d'un filet épervier de maille $1 \mathrm{~cm} \times 1 \mathrm{~cm}$.

Au laboratoire, les hôtes ont d'abord été examinés à l'œil nu et à la loupe binoculaire de marque Olympus BO 61 en vue de la recherche d'éventuels kystes sur le corps et les nageoires. Après dissection, tous les organes du poisson ont été prélevés individuellement et examinés. Le tube digestif a été dilacéré dans une boîte de pétri contenant de l'eau distillée. Les kystes trouvés ont été prélevés à l'aide des pinces fines et leur contenu examiné à l'objectif $100 \times$ du microscope. Les dessins des spores fraîches ont été exécutés à la chambre claire d'un microscope Wild M-20. Les mensurations ont été effectuées sur au moins 50 spores frâtches, à l'aide d'un micromètre oculaire, selon la technique proposée par Lom et Arthur (1989). Les frottis permanents des spores ont été fixés au méthanol, colorés selon la méthode panchromatique de MayGrünwald-Giemsa et montés au baume de Canada. Les microphotographies des spores (fraîches et colorées) ont été réalisées à l'aide du microscope Olympus $\mathrm{CH}$ 2, équipé d'un dispositif électronique de microphotographie.

\section{RÉSULTATS}

\section{THELOHANELLUS NIINEI N. SP. (figures 1-3, 10)}

Forme végétative : les trophozoïtes n'ont pas été trouvés, les spores sont diffuses dans la muqueuse intestinale.

Spores : les spores sont régulièrement ovoïdes (vues de face), avec un pôle antérieur étroit (figures 1, 10). Le plus grand diamètre s'observe au niveau du tiers postérieur de la spore (figures 1, 2). Les valves sont lisses. La capsule polaire est ovoïde et s'ouvre au pôle antérieur de la spore (figures 1-3, 10). Elle occupe un peu plus du tiers de la longueur de la spore. Le filament polaire est enroulé sur sept à huit tours de spire (figure 10a). Le sporoplasme, en forme de croissant, est très développé; il contient souvent une vacuole iodophile de forme et de taille variables (figures 1, 10a, 10b).

Mensurations : longueur : 14,6 (13-16) $\mu \mathrm{m}$; largeur : $8,3(7,3-9) \mu \mathrm{m}$; capsule polaire : 5,7 (5-7) $\times 3,6$ (33,8) $\mu \mathrm{m}$.

Hôte type : Schilbe mystus (Linné, 1758) (Schilbeidae). Localité : Edéa (sur la rivière Lem bass, affluent de la Sanaga) au Cameroun (Afrique centrale).

Organe parasité : intestin.

Prévalence : huit poissons parasités sur 20 examinés.

TheLOHANELLUS LAGDOENSIS N. SP. (figures 6-9, 11)

Forme végétative : dans la paroi intestinale de l'hôte, cette Myxosporidie forme des kystes blanchâtres, ovoïdes et de taille réduite $(140 \times 70 \mu \mathrm{m}$ en moyenne) . Ils sont polysporés.

Spores : de taille réduite ( 8 à $9 \mu \mathrm{m}$ de long), les spores sont piriformes avec une extrémité antérieure plus étroite, la postérieure étant large et arrondie (figures 6-7, 11). Les valves sont uniformément épaisses et lisses. La capsule polaire est située dans la moitié antérieure de la spore. Peu développée $(2,6 \times 1,8 \mu \mathrm{m}$ en moyenne), elle présente une sorte de "cou" caractéristique qui la relie au pôle antérieur de la spore (figures 6-9). Sur frottis frais, les spires du filament polaire ne sont pas apparentes au sein de la capsule polaire (figures 6-8). Le sporoplasme est développé et contient souvent une vacuole iodophile de forme et de position variables (figures 7-8, 11).

Mensurations : longueur : 8,4 (8-9) $\mu \mathrm{m}$; largeur : 4,3 $(3,8-5) \mu \mathrm{m}$; capsule polaire : 2,6 (2-3) × 1,8 (1,6-2) $\mu \mathrm{m}$; longueur du "cou" : 1-2 $\mu \mathrm{m}$.

Hôte type : Citharinus citharus (Geoffroy Saint-Hilaire, 1809) (Citharinidae).

Localité : Lagdo (dans la retenue de Lagdo sur la Bénoué) au Cameroun (Afrique centrale).

Organe parasité : intestin.

Prévalence : sept poissons parasités sur 20 examinés.

Thelohanellus CITHARINI Kostoingué, Fall, Faye ET Toguebaye, 1999 (figures 4-5, 12)

Forme végétative : dans la paroi intestinale, ce parasite développe de nombreux kystes subsphériques. Les pansporoblastes sont disporés.

Spores : les spores sont piriformes et allongées (trois fois plus longues que larges), avec une extrémité antérieure effilée (figures 4, 12). La capsule polaire est allongée (trois fois et demie plus longue que large), et s'ouvre au pôle antérieur de la spore (figures 4-5, 12). Sur les spores fraîches, on peut compter une dizaine de tours de spire du filament au sein de la capsule polaire (figure 12). Le sporoplasme est très développé (figures 4, 12).

Mensurations : longueur : 13,2 (11,5-14) $\mu \mathrm{m}$; largeur : $4,1(3,7-5) \mu \mathrm{m}$; capsule polaire : 6,7 (6-7,3) × 1,9 $(1,8-$ 2) $\mu \mathrm{m}$. 

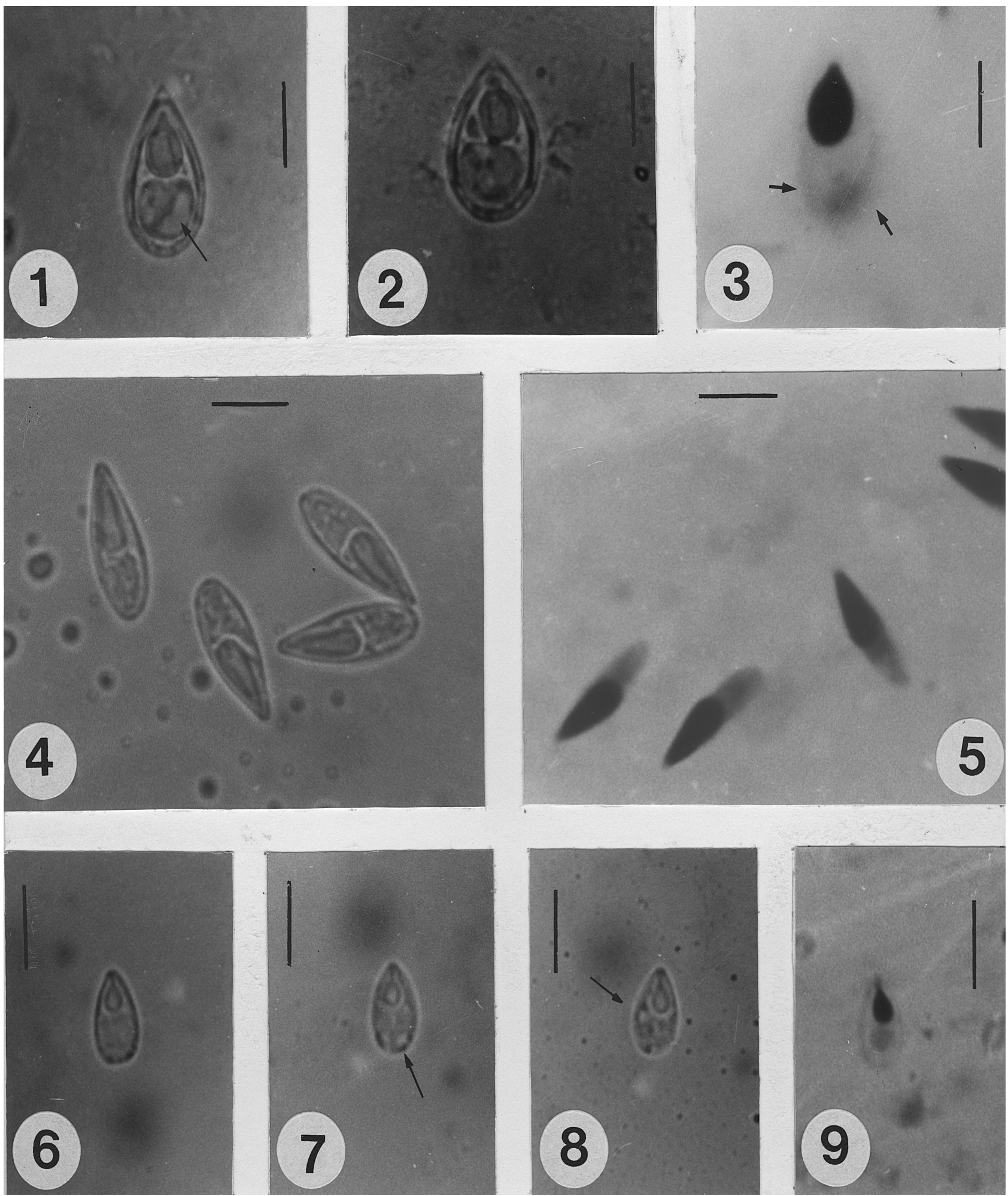

Figures 1-9. - Microphotographies des spores des différentes espèces de Myxosporidies étudiées. 1-3. Thelohanellus njinei n. sp. 1) spore fraîche, observer la vacuole iodophile (flèche), échelle : 6,5 $\mu \mathrm{m} ; 2$ ) spore fraîche montrant un sporoplasme très développé, échelle : 8,5 $\mu \mathrm{m}$; 3) spore colorée au MayGrünwald-Giemsa, échelle : 9,5 $\mu \mathrm{m}$.

4-5. Thelohanellus citharini Kostö̈ngué et al., 1999. 4) spores fraîches, échelle : 7,5 $\mu \mathrm{m}$; 5) spores colorées au MayGrünwald-Giemsa, échelle : $7,5 \mu \mathrm{m}$.

6-9. Thelohanellus lagdoensis n. sp. 6) spore fraîche, observer la capsule polaire avec son "cou" caractéristique, échelle : 7,5 $\mu \mathrm{m} ; 7)$ spore fraîche montrant une vacuole iodophile, échelle : 7,5 $\mu \mathrm{m}$; 8) spore fraîche, observer la position variable de la vacuole iodophile, échelle : $7,5 \mu \mathrm{m}$; 9) spore colorée au MayGrünwald-Giemsa, échelle : 7,5 $\mu \mathrm{m}$. 


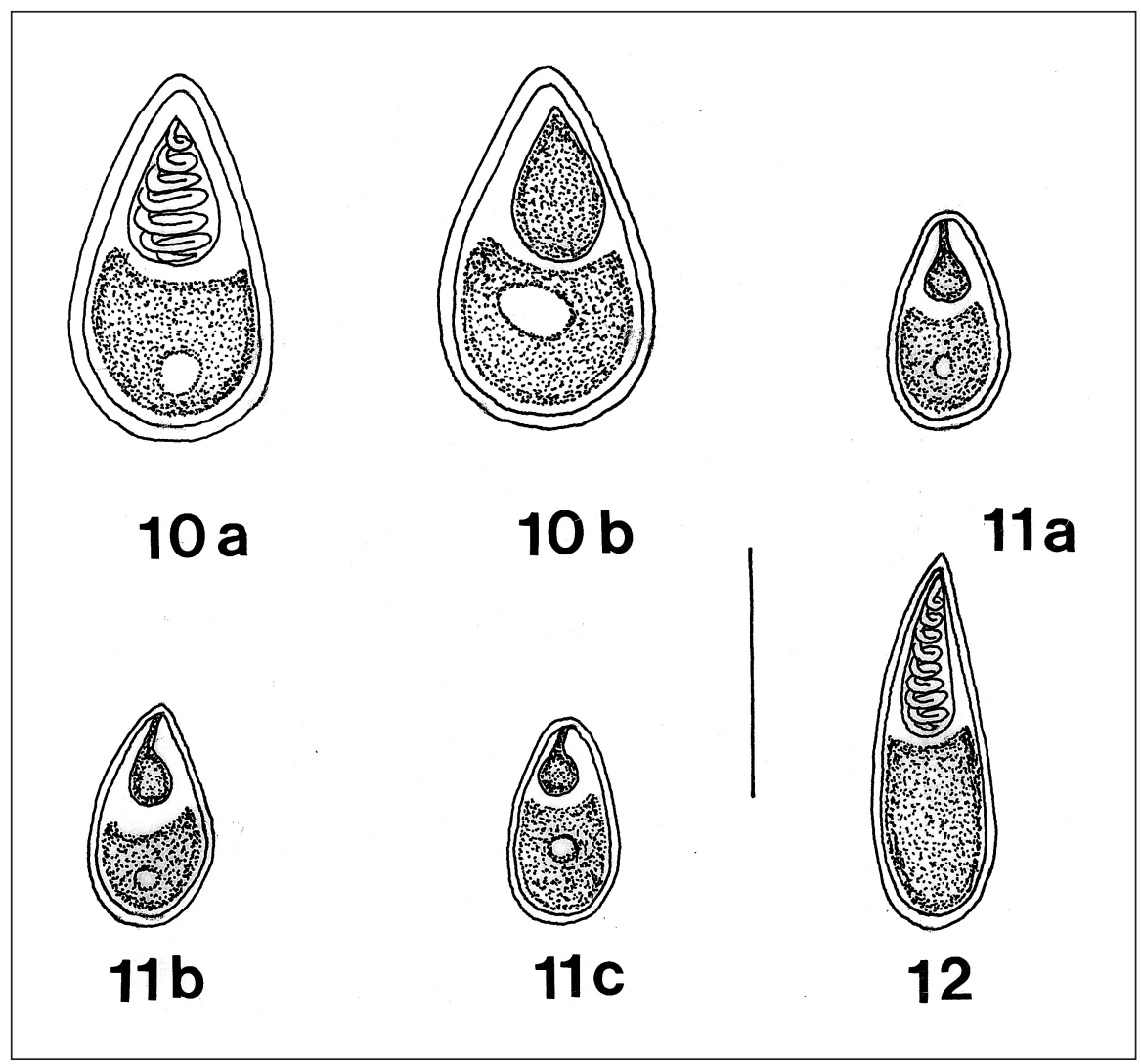

Figures 10-12. - Spores des Myxosporidies étudiées (échelle : $10 \mu \mathrm{m}$ ).

10. Thelohanellus njinei $\mathrm{n}$. sp. a) noter la forme générale de la spore et le nombre de tours de spire du filament au sein de la capsule polaire; b) le sporoplasme est granuleux.

11. Thelobanellus lagdoensis n. $\mathrm{sp}$. a) noter la forme générale de la spore; b et c) la capsule polaire présente un "cou" caractéristique.

12. Thelohanellus citharini Kostoïngué et al., 1999 : la spore est piriforme et allongée ; le sporoplasme est très développé.

Hôte type : Citharinus citharus (Geoffroy Saint-Hilaire, 1809) (Citharinidae).

Localité : Lagdo (dans la retenue de Lagdo sur la Bénoué) au Cameroun (Afrique Centrale).

Organe parasité : intestin.

Prévalence : quatre poissons parasités sur 20 examinés.

\section{DISCUSSION}

\section{THELOHANELLUS NJINEI N.SP.}

1 $\checkmark$ rois espèces de Thelohanellus ont déjà été décrites chez les Téléostéens d'eau douce au Cameroun : Thelohanellus valeti Fomena et Bouix, 1987, T. sanagaensis et T. assambai (Fomena et Bouix, 1994). T. valeti, parasite des Cyprinidae du genre Barbus, forme des spores plutôt piriformes et allongées (environ 2,7 fois plus longues que larges), avec une extrémité antérieure effilée et parfois recourbée. T. sanagaensis, parasite des branchies et des nageoires chez Labeo sp., forme des spores ellipsoïdales avec l'unique capsule polaire disposée obliquement par rapport au grand axe de la spore (caractère constant) et contenant neuf à 11 tours de spire du filament. Ces spores sont moins longues (11,6 um en moyenne) comparées à celles de l'espèce que nous décrivons. La forme générale de nos spores rappelle celle de $T$. assambai, para- site des branchies et des nageoires chez Labeo sp. Cependant, les spores décrites chez les Labeo sont nettement moins développées $(10,8 \times 5,5 \mu \mathrm{m})$, comparées à celles trouvées chez Schilbe mystus.

Thelohanellus taguii Fomena et al. 2004, parasite de nombreux organes chez Citharinus citharus au Tchad. Cette espèce se distingue par ses spores piriformes et très allongées (3,6 fois plus longues que larges), très développées (20,9 $\mu \mathrm{m}$ de long en moyenne) et contenant 16 à 20 tours de spire du filament au sein de la capsule polaire.

Thelohanellus bicornei Kabré et al., 2002 est un parasite des branchies chez Labeo coubie au Burkina-Faso. Bien que de dimensions comparables $(13,5 \times 8,4 \mu \mathrm{m}$ en moyenne), les spores de $T$. bicornei présentent deux éperons caractéristiques sur la grande valve. Thelohanellus costeae Sakiti, 1997, parasite branchial chez Labeo senegalensis au Bénin, forme des spores ovoïdes mais moins développées $(9,4 \times 5,6 \mu \mathrm{m})$, de même on note la présence d'un éperon sur l'une des valves. Thelohanellus sangibi Sarkar et Ghosh, 1990, parasite de Mystus guleo en Inde, bien que formant des spores ovoïdes, présente une capsule polaire moins développée $(4,5 \times 4 \mu \mathrm{m})$ et ne contenant que quatre à cinq tours de spire du filament. La taille réduite des spores $(10,9 \times 6,5 \mu \mathrm{m})$, la présence de trois à quatre stries sur les valves, éloignent $T$. bengalensis Sarkar et Choudhury, 1986 de l'espèce que nous décrivons. Theloha- 
nellus boggoti Qadri, 1962, (parasite de Labeo boggot en Inde), T. wallagoi Sarkar, 1985 (parasite de Wallago attu en Inde), diffèrent de la Myxosporidie de Schilbe mystus par de nombreux aspects (taille réduite des spores, nombre réduit de tours de spire du filament polaire, présence d'une troncature apicale, capsule polaire présentant une sorte de "cou" caractéristique).

Nous pensons que le parasite de Schilbe mystus est nouveau et proposons de le nommer Thelohanellus njinei $\mathrm{n}$. sp., en témoignage de sympathie pour le professeur Thomas Njiné de la Faculté des Sciences de l'Université de Yaoundé I.

\section{TheLOHANELLUS LAGDOENSIS N. SP.}

Des neuf espèces de Thelohanellus antérieurement décrites chez les poissons d'eau douce d'Afrique, le présent parasite ne peut être comparé qu'à Thelohanellus costeae Sakiti, 1997 (les autres espèces formant des spores nettement plus grandes). T. costeae forme en effet des kystes entre les filaments branchiaux et sur l'arc osseux chez Labeo senegalensis au Bénin. Les spores de cette espèce, bien que de dimensions comparables $(9,4 \times 5,6 \mu \mathrm{m})$, présentent une capsule polaire plus développée $(4-5,5 \times 2-3 \mu \mathrm{m})$ et qui contient 11 tours de spire du filament. De même on note la présence d'un appendice de 1,5 à $2 \mu \mathrm{m}$ de long sur la valve qui porte l'orifice de dévagination du filament polaire (caractère constant). La littérature fait état de la présence d'une sorte de "cou" caractéristique sur les spores de Thelohanellus boggoti Qadri, 1962. Les spores de cette espèce, bien que ovoïdes, sont plus grandes (11-12 × 6-7,5 $\mu \mathrm{m})$ comparées à celles que nous décrivons; de même, sa capsule polaire est plus développée $(5,5-7 \times 3,6-4 \mu \mathrm{m})$.

Thelohanellus wallagoi Sarkar, 1985, montre des spores piriformes et de dimensions réduites $(9,2 \times 4,8 \mu \mathrm{m}$ en moyenne), mais avec une troncature apicale et une capsule polaire nettement plus grande $(5,4 \times 2,7 \mu \mathrm{m}$ en moyenne).

Le parasite de Citharinus citharus qui se démarque des autres espèces connues (notamment par la taille réduite de ses spores et la présence d'une sorte de "cou" caractéristique au niveau de la capsule polaire) est nouveau. Nous proposons de le nommer Thelohanellus lagdoensis n. sp., du nom de la retenue de Lagdo dans laquelle les hôtes ont été capturés.

\section{Thelohanellus citharini Kostoingué, Fall,} Faye et Toguebaye, 1999

En 1999, Kostoïngué et al. ont décrit Thelohanellus citharini, parasite qui forme de volumineux kystes (1,5 mm de diamètre) dans le tissu cardiaque de Citharinus citharus au Tchad. La morphologie des spores que nous décrivons, l'hôte, correspondent aux données sur cette espèce. Bien que les spores présentées par Kostoïngué et al. soient moins longues $(11,1 \mu \mathrm{m})$, plus larges $(6,1 \mu \mathrm{m})$, et que ces auteurs n'aient observé que quatre à cinq tours de spire du filament au sein de la capsule polaire, nous pensons être en présence de la même espèce . La formation des kystes dans la paroi intestinale, la formation des spores plus longues (11,5$14 \mu \mathrm{m}$ ) et contenant une dizaine de tours de spire du filament polaire, sont des éléments à ajouter à la diagnose de cette Myxosporidie qui semble spécifique des Citharinidae.

\section{CONCLUSION}

T e genre Thelohanellus Kudo, 1933 regroupe aujourd'hui une cinquantaine d'espèces décrites à tra$\checkmark$ vers le monde, presque toutes histozoïques chez les poissons d'eau douce. L'unique forme coelozoïque connue (Thelohanellus wallagoi) a été décrite dans la vésicule biliaire de Wallago attu en Inde (Sarkar, 1985). Si en 1997, Fomena et Bouix, dans une clé de détermination des Myxosporidies des poissons d'Afrique ne recensaient que quatre espèces de Thelohanellus, ce genre est aujourd'hui représenté par neuf espèces chez les Téléostéens d'eau douce sur le continent. Les espèces décrites l'ont été chez les poissons capturés dans les pays suivants : Cameroun, Tchad, Bénin, Burkina-Faso et Égypte. La description de Thelohanellus njinei et Thelohanellus lagdoensis porte à 11 le nombre d'espèces du genre connues chez les poissons d'eau douce d'Afrique. Toutes les espèces africaines sont histozoïques et forment des kystes de taille variable dans divers organes chez leur(s) hôte(s) (branchies, nageoires, paroi stomacale, paroi intestinale, peau, cour, foie, opercules). Les branchies restent cependant l'organe préférentiel pour ces parasites particuliers (avec 63,63\% d'espèces récoltées). Les hôtes jusqu'à présent recensés appartiennent aux familles des Cyprinidae (sept espèces de Thelohanellus présentes), Citharinidae (trois espèces décrites) et Schilbeidae (une espèce). Au sein des Cyprinidae, le genre Labeo porte six espèces parasites (T. assambai, T. sanagaensis, T. ndjamenaensis, T. costeae, T. niloticus, T. bicornei). Chez les Citharinidae, Citharinus citharus à lui seul porte trois espèces (T. citharini, T. taguii, et T. lagdoensis). Ces observations confirment les remarques de Fomena et al., 2004 qui recensaient déjà chez cet hôte dix espèces de Myxosporidies appartenant aux genres Myxobolus, Henneguya, Parahenneguya, et Thelohanellus. Citharinus citharus ainsi que les Cyprinidae du genre Labeo semblent ainsi constituer des biotopes particulièrement favorables au développement des Myxosporidies.

Thelohanellus citharini Kostoïngué et al., 1999, parasite initialement décrit au Tchad a été retrouvé chez son hôte d'origine au Cameroun. La diagnose de cette 


\begin{tabular}{|c|c|c|c|c|}
\hline Espèce parasite & Hôte(s) & $\begin{array}{l}\text { Organe(s) } \\
\text { parasité(s) }\end{array}$ & Pays & Références \\
\hline $\begin{array}{l}\text { T. niloticus } \text { (syn }=\text { Myxobolus } \\
\text { unicapsulatus) }\end{array}$ & Labeo niloticus & Peau & Égypte & Gurley, 1893 (1) \\
\hline T. valeti & Barbus aspilus B. jae & $\mathrm{Br}+\mathrm{Es}+\mathrm{Mu}+\mathrm{Ma}$ & Cameroun & Fomena \& Bouix, 1987 (2) \\
\hline T. assambai & Labeo sp. & $\mathrm{Br}+\mathrm{Na}$ & Cameroun & Fomena et al., 1994 (3) \\
\hline T. sanagaensis & Labeo sp. & $\mathrm{Br}+\mathrm{Na}$ & Cameroun & Fomena et al., 1994 (3) \\
\hline T. costeae & Labeo senegalensis & $\mathrm{Br}$ & Bénin & Sakiti, 1997 (4) \\
\hline T. citharini & Citharinus citharus & Coeur & Tchad & Kostö̈ngué et al., 1999 (5) \\
\hline T. ndjamenaensis & Labeo parvus & $\mathrm{Br}$ & Tchad & Kostö̈ngué et al., 1999 (5) \\
\hline T. bicornei & Labeo coubie & $\mathrm{Br}$ & Burkina-Faso & Kabré et al., 2002 (6) \\
\hline T. taguii & Citharinus citharus & $\mathrm{Br}+\mathrm{Foie}+\mathrm{Op}+\mathrm{Int}$ & Tchad & Fomena et al., 2004 (7) \\
\hline
\end{tabular}

$\mathrm{Br}$ : branchie; Es : estomac; Int : intestin; Ma : mâchoire; Mu : muscle; Na : nageoire; Op : opercule.

Tableau I. - Liste systématique des différentes espèces de Thelohanellus antérieurement décrites chez les Téléostéens d'eau douce d'Afrique.

\begin{tabular}{|c|c|c|c|c|c|}
\hline $\begin{array}{l}\text { Espèces } \\
\text { parasites }\end{array}$ & Références & $\begin{array}{l}\text { Dimensions de la spore } \\
\qquad(\mathrm{L} \times \mathrm{l})\end{array}$ & $\begin{array}{l}\text { Dimensions de la capsule polaire } \\
\qquad\left(L^{\prime} \times l^{\prime}\right)\end{array}$ & nfp & n. ép \\
\hline T. niloticus & (1) & $5 \times 3,5$ & - & - & _ \\
\hline T. valeti $\quad$ type I & (2) & $12,0(11-13) \times 4,7(4-5.2)$ & $6,2(5,5-7) \times 2,3(1,8-3)$ & $6-8$ & - \\
\hline type II & (2) & $15,5(13,5-19,6) \times 5,4(4-6,9)$ & $7,7(6-9,2) \times 2,7(2-3,5)$ & $7-10$ & _- \\
\hline T. assambai & (3) & $10,8(9-12) \times 5,5(5-7)$ & $6,3(5-7,6) \times 3,4(3-4)$ & $5-6$ & _ \\
\hline T. sanagaensis & (3) & $11,6(10,5-13,5) \times 9,0(8,2-10,5)$ & $5,8(5-6,5) \times 3,7(3-4,1)$ & $9-11$ & - \\
\hline T. costeae & (4) & $9,4(8,5-10,5) \times 5,6(5-6,5)$ & $4,8(4-5,5) \times 2,6(2-3)$ & 11 & $\overline{1}$ \\
\hline T. bicornei & (6) & $13,5(13-14) \times 8,4(8-9)$ & $7,2(6,5-8) \times 3,7(3,5-4)$ & 10 & 2 \\
\hline T. citharini & (5) & $11,1(10-12) \times 6,1(6-7)$ & $6,6(6-7) \times 3,2(3-4)$ & $4-5$ & - \\
\hline T. ndjamenaensis & (5) & $10,3(10-11) \times 7,3(7-8)$ & $4,2(4-5) \times 3,2(3-5)$ & $4-5$ & - \\
\hline T. taguii & (7) & $20,9(19-23) \times 5,8(5-6,5)$ & $13,7(12-16) \times 3,2(2,5-3,5)$ & $16-20$ & - \\
\hline T. njinei n. sp. & $\mathrm{p}$ & $14,6(13-16) \times 8,3(7,3-9)$ & $5,7(5-7) \times 3,6(3-3,8)$ & $7-8$ & - \\
\hline T. lagdoensis n. sp. & $\mathrm{p}$ & $8,4(8-9) \times 4,3(3,8-5)$ & $2,6(2-3) \times 1,8(1,6-2)$ & - & - \\
\hline
\end{tabular}

nfp : nombre de tours de spire du filament; n.ép : nombre d'éperons; $\mathrm{p}$ : présent travail; les chiffres indiquant les références renvoient au tableau I.

Tableau II. - Mensurations (en mm) des spores des différentes espèces de Thelohanellus connues chez les poissons d'eau douce d'Afrique.

Myxosporidie doit être complétée avec des caractères nouveaux : formation des spores de taille très variable et pouvant être plus grande $(13,2 \mu \mathrm{m}$ de long en moyenne) ; formation d'une dizaine de tours de spire du filament au sein des capsules polaires; formation des kystes dans la paroi intestinale.

Thelohanellus lagdoensis n. sp., de par la taille réduite de ses spores $(8,4 \mu \mathrm{m}$ de long), figure parmi les plus petites spores connues dans ce genre à travers le monde. C'est la première fois qu'une Thelohanellus est décrite chez Schilbe mystus (Schilbeidae).

Avec la description de deux espèces nouvelles et la découverte de T. citharini (parasite jusqu'à présent connu au Tchad) chez son hôte d'origine, la faune des Myxosporidies du genre Thelohanellus décrites chez les poissons d'eau douce du Cameroun est portée à six, correspondant à 54,54\% d'espèces connues chez les Téléostéens d'eau douce d'Afrique et à environ $12 \%$ de la faune des Thelohanellus connues dans le monde. Les Cyprinidae et les Citharinidae constituent des hôtes de prédilection pour les Myxosporidies du genre Thelohanellus. Une synthèse sur les caractéristiques des spores des différentes espèces de Thelohanellus décrites en Afrique, leurs hôtes et les organes parasités est donnée dans les tableaux I et II.

\section{RÉFÉRENCES}

AbaKaR-Ousman. Les Myxosporidies (Myxozoa : Myxosporea) parasites des poissons d'eau douce du Tchad : faunistique et biologie des espèces inféodées à Oreochromis niloticus (Linné, 1758) et Sarotherodon galilaeus (Linné, 1758) (Cichlidae). Thèse de doctorat/Ph. D., Université de Yaoundé I, 2006, 163 p.

Bilong Bilong C.F., Tombi J., Nack J. \& Fomena A. Les parasites peuvent-ils être une cause de la réduction de la biodiversité des poissons ? Biosciences proceedings, 1998, 5, 113-119.

Ching H.L. \& MUnday D.R. Geographic and seasonal distribution of infectious stage of Ceratomyxa shasta Noble, 1950, a Myxozoan salmonid pathogen in the Fraser River system. Canadian Journal of Zoology, 1984, 62, 1075-1080.

Fall M., Fomena A., Kostoingue B., Diebakate C., Faye N. \& Toguebaye B.S. Myxosporidies (Myxozoa : Myxosporea) 
parasites des poissons Cichlidae du Cameroun, du Sénégal et du Tchad, avec la description de deux nouvelles espèces. Annales des Sciences Naturelles, 2000, 21, 81-92.

Fomena A. Contribution à l'étude des Myxosporidies (Protozoa : Myxozoa) parasites des poissons d'eau douce du Sud-Cameroun : systématique, ultrastructure, relations hôteparasite. Thèse de doctorat de $3^{\text {ème }}$ cycle, Université de Yaoundé, Cameroun, 1986, 276 p.

Fomena A. Myxosporidioses et Microsporidioses des poissons d'eau douce du Sud-Cameroun : étude faunistique, ultrastructure et biologie. Thèse de doctorat d'État, Université de Yaoundé I, 1995, 397 p.

Fomena A. \& Boutx G. Contribution à l'étude des Myxosporidies des poissons d'eau douce du Cameroun. III. Espèces nouvelles des genres Henneguya et Thelohanellus. Revue de Zoologie Africaine, 1987, 101, 43-53.

Fomena A. \& Bouix G. Myxosporea (Protozoa: Myxozoa) of the freshwater fishes in Africa: keys to the genera and species. Systematic Parasitology, 1997, 37, 161-178.

Fomena A., Marquès A., Bouix G. \& Njine T. Myxobolus bilongi n. sp., Thelohanellus assambai n. sp. et T. sanagaensis n. sp., Myxosporidies parasites de Labeo sp (Téléostéen : Cyprinidae) du bassin de la Sanaga au Cameroun (Afrique centrale). Annales de la Faculté des Sciences, HS $n^{\circ}$ 3, chimie, sciences naturelles, 1994, 131-142.

Fomena A., Abakar-Ousman., Ngassam P. \& Bouix G. Description de trois espèces nouvelles de Myxosporidies (Myxozoa : Myxosporea) parasites de Citharinus citharus (Geoffroy Saint-Hilaire, 1809) (Citharinidae) au Tchad (Afrique centrale). Parasite, 2004, 11, 83-88.

GuRley H.R. On the classification of the Myxosporidia, a group of Protozoan parasites infesting fishes. Bulletin of the United States Fisheries Commission, 1893, 11, 407-431.

Kabre G. B., Sakiti N. G., Marquès A. \& Sawadogo L. Thelohanellus bicornei n. sp. Myxosporidia (Myxosporea: Bivalvulida) a gill parasite of Labeo coubie Ruppel, 1832 (Osteichthyen, Cyprinidae) from Burkina-Faso, West Africa. Parasite, 2002, 9, 219-223.

Kent M.L., Andree K.B., Bartholomew J.L., El Matbouli M., Desser S.S., Devlin R.H., Feist S.W., Hedrick R.P., Hoffman R.W., Khattra J., Hallet S. L., Lester J.G., Longshaw M., Palenzeula O., Siddal M.E., \& XIaO C. Recent advances in our knowledge of the Myxozoa. Journal of Eukaryotic Microbiology, 2001, 48, 395-413.

Kostoïngue B., Fall M., Faye N. \& Toguebaye B.S. Three new myxosporidian (Myxozoa: Myxosporea) parasites of freshwater fishes from Chad (central Africa). Acta Protozoologica. 1999, 38, 323-326.

Lom J. \& Dykova I. Myxosporidia (Phylum Myxozoa), in: Protozoan parasite of fishes. Elsevier. Amsterdam-London-New York-Tokyo (Ed). Developments in Aquaculture and Fisheries Science, 1992, 26, 159-235.

LOM J. \& ARTHUR J.R. A guideline for the preparation of species descriptions in Myxosporea. Journal of Fish Diseases, 1989, 12, 151-156.

Okaeme A.N., Obiekezie A.I., Lehman J., Antai E.E., Madu C.T. Brief communications. Parasites and diseases of cultured fish of lake Kainji area Nigeria. Journal of Fish Biology, 1988, 32, 479-481.
QADRI S.S. On a new Myxosporidian Thelohanellus boggoti n. sp. from an Indian freshwater fish Labeo boggot. Arch. Protistenk, 1962, 106, 218-222.

SAKITI G.N. Myxosporidies et Microsporidies des poissons du Bénin : faunistique, ultrastructure, biologie. Thèse de doctorat d'État, Université nationale du Bénin, 1997, 296 p.

SARKAR N.K. Some coelozoic Myxosporidians (Myxozoa: Myxosporea) from freshwater fish of river Padma. Acta Protozoologica, 1985, 24 (1), 47-53.

SARKAR N.K. \& CHOUDHURY S.R. Thelohanellus bengalensis sp. $\mathrm{n}$. and Myxidium mystusium sp. n. (Myxozoa): two Myxosporidia from Indian freshwater Teleost. Acta Protozoologica, 1986, 25 (3), 359-362.

SARKAR N.K. \& GHosh S. Two new Myxozoan parasites of the genus Thelohanellus kudo 1933 (Myxosporea: Myxobolidae) from freshwater fishes of West Bengal, India. New Agriculturist, 1990, 1 (1), 35-38.

Reçu le 6 novembre 2006 Accepté le 14 février 2007 